\title{
Parlando, Mündlichkeit und neue Medien \\ Anmerkungen aus linguistischer Sicht
}

\section{Christa Dürscheid}

Im Beitrag werden zunächst die Bezeichnungen konzeptionell mündlich, medial mündlich und gesprochensprachlich voneinander abgegrenzt und die sprachlichen Merkmale von solchen Texten beschrieben, die unter das Stichwort Parlando fallen und als Kennzeichen eines neuen, an der Mündlichkeit angelehnten Schreibstils gelten (vgl. Sieber, 1998). Dann steht die Frage im Mittelpunkt, ob es gerechtfertigt ist, die private, textbasierte Kommunikation in den neuen Medien (z.B. E-Mail, SMS, Chat) als verschriftete Mündlichkeit zu charakterisieren und sie in die Nähe von Gesprächen zu rücken. Dabei lege ich den Schwerpunkt auf die Betrachtung von SMS- und Chattexten. Anhand solcher Texte wird gezeigt, dass sich in der privaten Schreibpraxis zahlreiche sprachliche Merkmale finden, die in der Linguistik als konzeptionell mündlich bezeichnet werden, die aber keineswegs mit dem gleichgesetzt werden dürfen, was Peter Sieber mit Blick auf Maturarbeiten als Parlando-Stil charakterisiert. Die Betrachtungsebenen müssen also sorgfältig getrennt werden, und es sollte aus linguistischer Sicht nur dann von Mündlichkeit in den neuen Medien gesprochen werden, wenn damit tatsächlich Gesprochensprachliches gemeint ist (also z.B. Internet-Telefonate via Skype). In allen anderen Fällen, so werde ich in meinem Beitrag abschliessend darlegen, sollte nur von konzeptioneller Mündlichkeit, nicht aber von Mündlichkeit oder Parlando die Rede sein.

\section{Mündlichkeit in aller Munde}

Im Titel des vorliegenden Beitrags steht das Wort Mündlichkeit im Zentrum und das nicht nur im wörtlichen, sondern auch im übertragenen Sinne. Damit wird an ein Thema angeknüpft, das derzeit sowohl in der Schule als auch in bildungspolitischen und fachdidaktischen Debatten eine wichtige Rolle spielt. So wird im Schulunterricht der mündlichen Kommunikation mehr Beachtung geschenkt, als dies noch in früheren Jahren der Fall war. Das zeigt sich unter anderem an dem Erfolg des Wettbewerbs Jugend debattiert, der in der Schweiz erstmals im Schuljahr 2005/2006 durchgeführt wurde und in den Schulen 
seither viel Resonanz findet. ${ }^{1}$ Auch in fachwissenschaftlichen Arbeiten wird die Frage, wie man die Sprech- und Gesprächskompetenz der Schülerinnen und Schüler fördern kann, zunehmend thematisiert (Roth, 2006), und es werden Lernszenarien vorgestellt, die das Argumentieren, Erzählen und Erklären im Unterricht, aber auch das Zuhören betreffen (Krelle \& Spiegel, 2009). Zudem gibt es interessante Informationsmaterialien für Lehrerinnen und Lehrer, die Hinweise zur Förderung der mündlichen Sprachkompetenz enthalten (Eriksson, 2010). Doch all dies ändert nichts an dem Umstand, dass der Bereich der Mündlichkeit lange Zeit ein Schattendasein führte. So heisst es im Vorwort zum Handbuch «Mündliche Kommunikation und Gesprächsdidaktik»: «Die letzten zwanzig Jahre haben uns einen Forschungsboom zum Erwerb der geschriebenen Sprache beschert ... . Eine analoge Entwicklung hat im Bereich des Mündlichen mit deutlicher Verzögerung eingesetzt» (Becker-Mrotzek, 2009, S. XIII). Diese Entwicklung hat nun aber tatsächlich eingesetzt. Das Thema ist heute nicht mehr nur für den Fremdsprachenunterricht von Bedeutung, sondern stellt auch in der Unterrichtsforschung zum Fach Deutsch und in der Diskussion um Kompetenzmodelle einen wichtigen Bereich dar (Eriksson, 2009). Nicht von ungefähr gibt es in dem oben erwähnten, von Michael Becker-Mrotzek (2009) herausgegebenen Handbuch mehrere Beiträge, die sich mit der Analyse mündlicher Kommunikation und der Entwicklung mündlicher Kommunikationskompetenz befassen und hierzu gute Überlegungen anstellen (z.B. Quasthoff, 2009).

Doch nicht nur in der Deutschdidaktik ist im Bereich der Mündlichkeit etwas in Bewegung geraten, auch in einer ihrer Bezugswissenschaften, in der Sprachwissenschaft, zeigt sich eine Neuorientierung. Wenn auch aus anderen Gründen, ${ }^{2}$ so galt doch auch für die Linguistik lange Zeit, dass die Forschungsperspektive schriftzentriert war und das Gesprochene, wie Johannes Schwitalla (2006) treffend feststellt, «im Vergleich zum Geschriebenen meist den Kürzeren» (S. 14) zog. Doch hat sich dies, so betont Schwitalla (2006) ebenfalls, seit den 1960er Jahren geändert. Heute stellen die Gesprächsanalyse, die GesprocheneSprache-Forschung und die Frage der Unterscheidung von Mündlichkeit und Schriftlichkeit wichtige Forschungsfelder in der Linguistik dar und werden auch in der Lehre gebührend thematisiert. Das sieht man an Einführungswerken, die in den letzten Jahrzehnten zum Thema erschienen sind (z.B. Brinker \& Sager, 1973/2010); das sieht man aber auch daran, dass es in der Duden-Grammatik, anders als dies in früheren Auflagen der Fall war, nunmehr ein separates Kapitel zur gesprochenen Sprache gibt (Duden, 2009, S. 1165-1244).

Ein Grund, warum das Thema Mündlichkeit in der Linguistik nun mehr Beachtung erfährt, ist sicher auch der, dass auch in der geschriebenen Sprache zunehmend Merkmale anzutreffen sind, die lange Zeit nur als charakteristisch für die gesprochene Sprache galten. Das resultiert aber nicht nur aus der geänderten Datenlage, es hängt auch damit zusammen, dass in der linguistischen Forschung auch solche Texte in den Blick genommen werden, die nicht litera- 
risch hochstehend sind. Das war in früheren Zeiten anders. So stellte der Sprachwissenschaftler Otto Behaghel in seinem noch heute viel zitierten Vortrag zum geschriebenen und gesprochenen Deutsch aus dem Jahre 1899 fest:

Wenn in den letzten Jahrhunderten von deutscher Sprache geredet wurde, wenn angesehene Gesellschaften der deutschen Sprache ihr Pflege widmeten, wenn Lehrgebäude und Wörterbücher der deutschen Sprache entstanden, so war es die vornehme, würdevolle, streng abgemessene Sprache der Schrift, des Buches, die man im Auge hatte, die Sprache derer, die vielbewundert auf den Höhen des Schriftums sich bewegten. (Behaghel, 1899/1927, S. 11)

Wie in diesem Zitat angedeutet, lag der Schwerpunkt in den Grammatiken und Lehrbüchern früherer Jahrhunderte zum einen auf der geschriebenen und nicht auf der gesprochenen Sprache, zum anderen wurde die "Sprache der Schrift» mit der hohen Sprache der Dichtung, der Literatursprache gleichgesetzt. In Ansätzen gilt dies noch bis heute. So kommt die Hochschätzung der geschriebenen Sprache dann zum Ausdruck, wenn davon die Rede ist, jemand spreche «wie gedruckt» bzw. jemand spreche «nach der Schrift».3 Schaut man sich Geschriebenes allerdings genauer an, stellt man rasch fest, dass in der Schrift häufig ein informeller Duktus anzutreffen ist, der der gesprochenen Sprache sehr nahe kommt. Das gilt nicht nur für Webseiten im Internet, das gilt auch für literarische Texte, in denen ein informelles, an die Mündlichkeit angelehntes Schreiben häufig als Stilmittel eingesetzt wird. Illustriert sei dies an einem Beispiel aus dem Roman «Dinge, die wir heute sagten», für den Judith Zander im Herbst 2010 den Deutschen Buchpreis erhielt. In dem Roman ist zu lesen: "Und nun haben wir zwei Autos und sind immer noch nicht zufrieden. Weil Friedhelm immer nur mit dem kleinen ollen Fiat zur Arbeit fahren muss, weil ich mit dem nicht klar komm, ich bin eben so an den Opel gewöhnt.» Diese Textpassage wurde in einem Artikel der Literaturkritikerin Iris Radisch in der ZEIT vom 4. Oktober 2010 zitiert und als Beispiel für den «Plapperton» in der deutschsprachigen Gegenwartsliteratur angeführt. Radisch schreibt dazu: «Diese Mündlichkeit - eigentlich von den Brüdern Grimm bis zu Kempowski eine Quelle literarischer Erneuerung - wirkt eher kleinlaut. Das Alltagssprachliche soll in diesen Beispielen Lebendigkeit nachstellen, die originale Tonspur der Wirklichkeit wiedergeben, und hat doch die gegenteilige Wirkung.»

Es ist hier nicht der Ort, den Schreibstil in der aktuellen Literatur weiter zu kommentieren; relevant für die hier angestellten Überlegungen ist das Folgende: Mündliches hält zunehmend Einzug in die Schriftlichkeit. So stellt auch Schwitalla (2006) fest, dass «in den letzten Jahrzehnten ... immer mehr Formen des Gesprochenen auch in schriftlichen Medien verwendet» würden und dass sich dies in Tageszeitungen, aber «noch stärker in den neuen elektronischen Medien» (S. 17) zeige. Allerdings stellt sich hier die Frage, ob diese Formulierungen den Sachverhalt korrekt beschreiben. Fakt ist, dass eine E-Mail oder eine SMS 
geschrieben wird, in welchem Sinne können diese Nachrichten also gesprochensprachlich sein? Das gilt auch für den Chat, eine schriftliche Konversation, die am Computer geführt werden kann, wenn die Beteiligten gleichzeitig im selben virtuellen Raum eingeloggt sind. Über die Chatkommunikation schreibt Kilian (2006), dass es sich dabei um «[m] ündlich entfaltete Schriftlichkeit» (S. 76) handle und die Schreiberinnen und Schreiber ihre Texte so konzipierten, «als befänden sie sich in einer nähesprachlichen Face-to-face-Gesprächssituation» (S. 75). An anderer Stelle bezeichnet der Verfasser diesen Schreibstil als "Chat,Parlando " und schliesst damit an einen Begriff an, den Peter Sieber bereits in den 1990er Jahren, also vor dem Einzug der neuen Medien, für eine spezifische, an der Mündlichkeit orientierte Art und Weise der Textgestaltung geprägt hat.

Damit ist die Brücke geschlagen zum zweiten Schlüsselwort im Titel des vorliegenden Beitrags, Parlando. Die folgenden Fragen drängen sich hier auf: Ist es berechtigt, die in den neuen Medien vorkommenden sprachlichen Phänomene, wie Kilian (2006) es tut, als «eine besondere Art, Parlando'» (S. 76) $\mathrm{zu}$ charakterisieren? Wo liegen die Unterschiede, wo die Gemeinsamkeiten zwischen Parlando und Mündlichkeit? Wie ist in diesem Kontext die gesprochene Sprache, wie das Gespräch zu verorten? Aus meiner Sicht sind hier einige begriffliche Präzisierungen notwendig. Dies wird Gegenstand des nächsten Abschnitts sein, bevor in Abschnitt 3 einige SMS- und Chattexte mit Auszügen aus einer Maturarbeit verglichen werden, um die Gemeinsamkeiten und Unterschiede im jeweiligen Schreibstil zu veranschaulichen. Der Beitrag schliesst mit Überlegungen zu der Frage, ob das von Jugendlichen in ihrer Freizeit häufig praktizierte Internet- und SMS-Schreiben möglicherweise einen Einfluss auf das Schreiben in der Schule hat.

\section{Gesprochene Sprache, Mündlichkeit, Gespräch und Parlando}

In diesem Abschnitt werden zunächst terminologische Fragen geklärt: 1) Ist die Bezeichnung gesprochene Sprache gleichzusetzen mit Mündlichkeit? 2) Wo liegt der Unterschied zwischen gesprochener Sprache und Gespräch? 3) Wo liegt der Unterschied zwischen Mündlichkeit und Parlando? 4) Wie stehen diese beiden Wörter in Relation zur Bezeichnung gesprochene Sprache, zumal sie diesen Aspekt ja auch im Wortlaut mittragen? Im Anschluss werde ich erläutern, welche Eigenschaften als charakteristisch für die gesprochene Sprache gelten und welche Eigenschaften nach Peter Sieber Kennzeichen für das Parlando-Schreiben sind. An dieser Stelle sei noch angemerkt, dass ein weiterer Terminus, der in der Diskussion um Mündlichkeit und Schriftlichkeit verwendet wird, in den weiteren Ausführungen unberücksichtigt bleibt: Oralität. Es sei dazu so viel gesagt: In der Linguistik wird Oralität häufig gleichgesetzt mit Gesprochensprachlichkeit bzw. mit Mündlichkeit. In sozial- und kulturwissenschaftlich ausgerichteten Arbeiten ist Oralität, wie die 
grundlegende Arbeit von Walter Ong (1982/1987) zeigt, auf einer anderen Ebene angesetzt. Hier unterscheidet man zwischen Oralität und Literalität respektive oralen und literalen Kulturen, wobei orale Kulturen solche sind, die vor der Entwicklung der Schrift existierten bzw. auch heute noch unberührt von der Schriftentwicklung sind (Dürscheid, 2006, S. 53).

Kommen wir nun zu den ersten beiden Fragen, zur Abgrenzung von gesprochener Sprache, Mündlichkeit und Gespräch: Um ein Gespräch handelt es sich, wenn eine Interaktion vorliegt, an der mindestens zwei Personen beteiligt sind, und wenn sich diese Interaktion in der gesprochenen Sprache vollzieht. Der Begriff gesprochene Sprache ist dagegen weiter gefasst. Er beinhaltet nicht nur dialogische, sondern auch monologische Sequenzen (z.B. Reden, Predigten, Vorträge), bezieht sich aber nur auf die phonische Seite der Sprache, auf die Lautung. Letzteres gilt für die Bezeichnung Mündlichkeit nicht: Hier unterscheidet man in der Linguistik zwischen konzeptioneller und medialer Mündlichkeit (vgl. Koch \& Oesterreicher, 1994). Nur mediale Mündlichkeit bezieht sich auf die phonische Realisierung von Sprache, konzeptionelle Mündlichkeit dagegen meint die in einer Äusserung gewählte Ausdrucksweise. Kommen in einem Text z.B. unvollständige Sätze (z.B. _ verstehe ich nicht), reduzierte Wortformen (z.B. ich hab_ _), umgangssprachliche Wörter (z.B. kriegen) oder Gesprächspartikeln (z.B. er ist halt ein Lügner) vor, dann wird er als konzeptionell mündlich bezeichnet. Dabei kann es durchaus sein, dass es in einem solchen Text Passagen gibt, die stärker konzeptionell mündlich sind, und andere, auf die das nicht zutrifft (z.B. in einem privaten Brief). Auch kommt es vor, dass eine Textsorte als Ganzes näher am Mündlichkeitspol verortet wird als eine andere. So ist anzunehmen, dass eine Postkarte aus den Ferien mehr Merkmale konzeptioneller Mündlichkeit aufweist als ein Geschäftsbrief, der zur ersten Kontaktaufnahme an einen Kunden geschickt wird. Dabei ist die konzeptionelle Dimension strikt von der medialen Dimension zu trennen. Charakterisiert man in der Linguistik eine Äusserung als medial mündlich bzw. komplementär dazu als medial schriftlich, so bezieht sich dies schlicht darauf, ob gesprochen bzw. geschrieben wird. Wird z.B. gesagt, dass ein Telefonat medial mündlich sei, heisst das nicht, dass es dies auch in konzeptioneller Hinsicht ist. Hier gibt es durchaus Unterschiede, die mit dem Anlass des Telefongesprächs zusammenhängen. Zwar kann man einwenden, dass die beiden Ebenen, medial mündlich und konzeptionell mündlich, in vielen Fällen ohnehin zusammenfallen und es deshalb oft genüge, das Attribut mündlich ohne weiteren Zusatz zu verwenden. Doch eben weil es Konstellationen gibt, in denen eine Äusserung medial mündlich, aber konzeptionell schriftlich ist (z.B. ein Plädoyer vor Gericht) oder umgekehrt (z.B. ein Feriengruss auf der Postkarte) - müssen die Ebenen getrennt werden. Dies zeigt sich auch, wenn man Nachrichten analysiert, die über Computer (z.B. als E-Mail) oder über das Handy (als SMS) verschickt wurden. ${ }^{4}$ Viele dieser Texte weisen Merkmale konzeptioneller Mündlichkeit auf (siehe dazu ausführlich den Sammelband von Schlobinski, 2006), es wäre aber 
verkürzt zu sagen, sie seien mündlich. Das gilt ebenso für die Bezeichnung gesprochensprachlich: Eine E-Mail ist nicht gesprochensprachlich, sie ist und bleibt schriftbasiert, auch wenn im Anhang Bilder, Videoclips oder Tondateien mitgeschickt werden können.

Das führt uns zu der Frage, worin die Unterschiede zwischen Gesprochensprachlichkeit und Schriftlichkeit bzw. Schriftbasiertheit, d.h. zwischen gesprochener und geschriebener Sprache liegen. An anderer Stelle habe ich diese Unterschiede bereits erläutert (Dürscheid, 2006, S. 24-34), deshalb sei hier nur so viel gesagt: Die gesprochene Sprache ist flüchtig, sie ist situationseingebunden, sie unterliegt den Bedingungen von Zeit und Raum und ist phylo- und ontogenetisch primär. Kommunikation in gesprochener Sprache verläuft synchron, Mimik, Gestik und Intonation treten zu den verbalen Ausdrucksmitteln hinzu. Weiter ist die gesprochene Sprache körpergebunden und stellt ein Lautkontinuum dar, besteht also nicht, wie die Schrift, aus einer Summe diskreter Einheiten. Nur die beiden letztgenannten Eigenschaften sind konstitutiv für gesprochene Sprache, in allen anderen Fällen handelt es sich um Merkmale, die durchaus auch wegfallen können. So gilt für ein Telefonat nicht, dass die Kommunikation im selben Raum erfolgt und situationseingebunden ist, auf die Situation kann also auch nicht mit einer deiktischen Äusserung vom Typ Das da gefällt mir Bezug genommen werden. Andererseits handelt es sich auch bei einem Telefonat - trotz räumlicher Distanz - um eine synchrone Kommunikation, die Produktion und die Rezeption der Äusserung fallen zusammen. So zeigen sich in einem Telefongespräch, wie in einem Face-to-Face-Gespräch, sprachliche Phänomene, welche der Tatsache geschuldet sind, dass die Formulierungen nicht vorgefertigt sind, sondern erst im Äusserungsprozess selbst, während des Telefonats, entstehen und in Abhängigkeit von der Hörerreaktion eine andere Richtung nehmen können. $\mathrm{Zu}$ diesen Phänomenen gehören Wort- und Konstruktionsabbrüche, Versprecher, Wortwiederholungen, Pausen und Verzögerungsphänomene wie $h m$ oder äh (Schwitalla, 2006, S. 88-90). Hinzu kommen syntaktische Konstruktionen, die im Gesprochenen weit verbreitet und nahezu unauffällig sind (z.B. die Konjunktion weil mit Verbzweitstellung oder Dativ-Possessiv-Konstruktionen vom Typ dem Vater sein Haus). Einige dieser Formulierungen treten zwar auch im Geschriebenen auf, aber in der Regel nur dann, wenn der Text in einem stark informellen, dialogischen Kontext steht, wie es z.B. in der Chatkommunikation der Fall ist (siehe dazu Abschnitt 3).

Damit kommen wir zum zweiten Fragenkomplex, zur Unterscheidung von gesprochener Sprache, Mündlichkeit und Parlando. Dem Parlando-Konzept widmet Peter Sieber 1998 eine fast 300 Seiten umfassende Arbeit, zehn Jahre später stellt er die wichtigsten Aspekte daraus nochmals in einem knappen Überblicksartikel zusammen (Sieber, 2008). Darin charakterisiert er das hinter dem Parlando-Schreiben stehende Textmuster folgendermassen: «Hier wird in die Schrift übernommen, was bis vor kurzem vor allem in die mündliche Kommunikation gehört hat: Direktheit, kurze Planungszeit, das Vertrauen auf 
die gemeinsam geteilte Kommunikationssituation» (Sieber, 2008, S. 281). Illustriert wird dieser Schreibstil an einem Maturaufsatz aus dem Jahr 1991, der in Sieber (1998, S. 32-34) in voller Länge nachzulesen und auch in Sieber (2008) in Auszügen abgedruckt ist. Der Schüler sollte einen Text zum Thema «Gesichter» schreiben. Sein Aufsatz beginnt mit den Worten «Wir stehen vor einer riesigen schwarzen Wand ... und warten. Weder Sie noch ich wissen, was wir gleich auf dieser Wand sehen werden, oder?». Sieber (1998) nennt mit Blick auf diesen Text die folgenden Parlando-Phänomene: Die Leserin/der Leser wird zu Beginn direkt angesprochen, gewissermassen an die Hand genommen ("Weder Sie noch ich wissen ...»), im weiteren Verlauf finden sich syntaktische Konstruktionen "mit grosser Nähe zur Sprechsprache» (S. 39), Unverbundenes steht nebeneinander, vieles wird nur implizit gesagt, häufig werden Auslassungspunkte gesetzt, wo man weitere Erläuterungen erwarten würde.

Alle diese Merkmale beziehen sich auf die Textoberfläche; dahinter aber zeigten sich, so Sieber, grundlegende Veränderungen in der Schriftlichkeit. Dieses Textmuster, das sich im schulischen Kontext erst in neueren Arbeiten finde, sei dadurch gekennzeichnet, dass die Schülerinnen und Schüler in ihren Texten eigene Erfahrungen und Erlebnisse thematisierten und dabei Direktheit, Authentizität und eine «starke Orientierung an der Mündlichkeit (insbesondere was Planung, Struktur und Oberfläche betrifft)» zu beobachten sei (Sieber, 1998, S. 150 f.). ${ }^{5}$ Selbstverständlich bleibt aber auch dieses Schreiben ein Schreiben, typische Eigenschaften gesprochener Sprache fehlen. Insofern wäre es falsch, das Parlando-Schreiben als mündlich zu bezeichnen. Es finden sich aber Ausdrucksweisen, die denen gleichen, welche nach Koch und Oesterreicher (1994) der konzeptionellen Mündlichkeit zuzuordnen sind. Nicht von ungefähr nimmt auch Peter Sieber auf ihre Unterscheidung von konzeptioneller und medialer Mündlichkeit bzw. Schriftlichkeit Bezug und erläutert, dass es hier Entsprechungen zum Parlando-Schreiben gebe. So stellt Sieber (1998) fest: "Wesentliche Kommunikationsbedingungen der konzeptionellen Mündlichkeit - wie Dialogizität, Vertrautheit mit dem Partner, freie Themenentwicklung, Affektivität - sind ebenfalls zentrale Merkmale von Parlando, auch wenn sie schriftlich - in veränderter Form realisiert werden müssen» (S. 191). Als Evidenz führt er zum einen an, dass in Parlando-Texten die Vertrautheit mit dem Partner durch die direkte Leser-Ansprache zu realisieren versucht werde, zum anderen die Dialogizität "durch eine fiktive Gesprächssituation evoziert» werde (Sieber, 1998, S. 191). Die Dialogizität entspricht aber, das sei kritisch angemerkt, nicht dem, was Koch und Oesterreicher (1994) darunter verstehen. Diese ist dadurch gekennzeichnet, dass die an der Kommunikation Beteiligten unmittelbar auf die Äusserungen reagieren können, dass eine direkte Interaktion stattfindet. Das ist bei einer Maturarbeit nicht der Fall, sie gehört, wie Kilian (2006, S. 75) zu Recht betont, zu den monologischen Textsorten. Auch andere, von Koch und Oesterreicher (2007, S. 351) genannte Parameter für kommunikative Nähe wie Privatheit, raumzeitliche Nähe und kommunikative Kooperation haben in einer 
Maturarbeit keine Entsprechung. Zwar kann man hier mit Peter Sieber geltend machen, dass diese Kommunikationsbedingungen im Parlando-Schreiben sprachlich «evoziert» werden; doch gerade darin liegt der wesentliche Unterschied: Die sprachlichen Mittel mögen solche Kommunikationsbedingungen evozieren, sie resultieren aber nicht daraus. Hinzu kommt, dass ein Schultext in einer anderen Erwartungshaltung rezipiert wird, als dies bei einem Text der Fall ist, der tatsächlich in einem dialogischen Kontext steht. In einer Maturarbeit kann die konzeptionelle Mündlichkeit meines Erachtens nur toleriert werden, wenn sie als gut eingesetztes Stilmittel erkannt wird. Wie ich im nächsten Abschnitt zeigen werde, darf auch aus diesem Grunde das konzeptionell mündliche Schreiben in den neuen Medien nicht mit dem Parlando-Schreiben in Maturaufsätzen gleichgesetzt werden. Es unterliegt sowohl auf der Produktionsals auch auf der Rezeptionsseite anderen Bedingungen.

\section{SMS- und Chattexte versus Maturaufsätze}

Um den Unterschied zwischen dem konzeptionell mündlichen Schreiben in den neuen Medien und dem Parlando-Schreiben in Schultexten deutlich zu machen, werden im Folgenden private SMS- und Chattexte mit Textpassagen aus dem oben erwähnten Maturaufsatz verglichen. Die Beispiele zum SMS-Schreiben stammen aus dem Schweizer SMS-Korpus (siehe dazu www.sms4science.ch), das insgesamt knapp 24'000 SMS aus allen Sprachregionen der Schweiz umfasst. Im Folgenden wurden nur solche Textnachrichten ausgewählt, die in Standarddeutsch geschrieben wurden, denn nur dann ist überhaupt ein Vergleich zwischen Sequenzen aus einer Maturarbeit und dem SMS-Schreiben möglich. Die nicht-dialektalen SMS stellen aber, das sei hier eigens betont, nur ca. $25 \%$ aller deutschsprachigen SMS ( $\left.\mathrm{n}=18^{`} 168\right)$ im SMS-Korpus dar. Das Alter der Schreiberinnen und Schreiber der Textnachrichten unter (4) bis (6) liegt zwischen 17 und 21 Jahren.

Auszüge aus dem Maturaufsatz (Sieber, 1998, S. 32-33)

(1) Ich möchte, dass Sie mich auf meinem Durchgang durch eine der besten wissenschaftlichen Ausstellungen begleiten ... .

(2) Und wir? Besitzen wir ebenfalls ein solches Charakterface?

(3) Ich schlage vor, wir lassen die medizinische Anatomie des Gesichtes aus, da wir sie in jedem Lexikon nachschlagen können und gehen direkt zur dritten grossen Abteilung hinüber .... .

Auszüge aus dem SMS-Korpus:

(4) koennen wir machen, wann und wo, passt dir am besten als tp?

(5) Wie gross ist die Datei. Ich hätte einen USB mit ca. Noch 3 GB hier.

(6) Weißt du tel. von mech. Werkstatt? 
Im Maturaufsatz wird die Dialogizität durch Formulierungen wie «Ich möchte, dass Sie ...», oder durch das Pronomen wir (z.B. «Besitzen wir.. «), das die Adressatin, den Adressaten einbezieht, hergestellt. Zudem wird in (3) ein Vorschlag gemacht, der sich direkt an die Leserin/den Leser richtet. Dies dient dazu, die Leserschaft gleichsam in den Text hineinzuholen und an dem Rundgang durch die Ausstellung teilhaben zu lassen. Es ist offensichtlich, dass der Schüler diese Formulierungen in seinem Aufsatz als Stilmittel verwendet; er weiss, dass er sich keineswegs in einer dialogischen Situation befindet, sondern einen Text verfasst, der für sich selbst sprechen muss und auf den er keine direkte Antwort bekommt.

Vergleichen wir damit die drei SMS in den Beispielen (4) bis (6). Diese Texte wurden in dem Bewusstsein geschrieben, dass die/der andere direkt auf die Nachricht antworten kann, dass die eigene Nachricht Teil einer Interaktion ist und dass auch deshalb vieles nur implizit gesagt werden muss. Das zeigt sich an der Nachricht (4), die vermutlich als Antwort auf eine zuvor erhaltene SMS verfasst wurde. Hier wird nicht gesagt, an welchen Vorschlag mit der Formulierung «koennen wir machen» angeknüpft wird, was gemacht werden kann. Selbst das Objektpronomen das, das einen Bezug zur vorangehenden Äusserung herstellen könnte, fehlt in dieser Sequenz. Aus syntaktischer Sicht liegt eine Ellipse vor, aus kommunikativer Sicht ist dies aber keineswegs der Fall, alle wichtigen Informationen werden gegeben. Der Bezugstext geht dem Schreiben ja voraus, er ist möglicherweise Sekunden vorher angekommen, auch verfügen die Beteiligten über ein gemeinsames Hintergrundwissen und sind vermutlich gut miteinander bekannt. Die Beteiligten wissen also, wovon die Rede ist. Das gilt auch für die Textnachrichten in (5) und (6), in denen jeweils eine Frage steht. Es ist zu vermuten, dass diese Fragen nicht erst Tage später beantwortet wurden. Doch auch wenn dies der Fall gewesen sein sollte: Die Texte wurden in dem Bewusstsein verfasst, dass unmittelbar darauf eine Antwort kommt. Das mag selbstverständlich scheinen, aus linguistischer Sicht ist es aber ein wichtiger Aspekt: Die Dialogizität wird nicht evoziert, sie ist vielmehr im Bewusstsein der Schreiberin/des Schreibers präsent. Mit anderen Worten: Eben weil die SMS anders als z.B. Briefe - nur minimal zeitversetzt hin und her wechseln können, werden sie oft so verfasst, als befände man sich in einem Gespräch. Daraus resultiert auf Produktionsseite eine konzeptionell mündliche Ausdrucksweise, auf Rezeptionsseite eine Toleranz gegenüber diesem informellen Schreiben.

Kommen wir zum nächsten Punkt des Vergleichs, zur Lexik, zur Syntax und zur Orthographie in den beiden Schreibkontexten. Die oben angeführten Passagen aus dem Maturaufsatz weisen keine lexikalischen Besonderheiten auf, an anderer Stelle kommen aber durchaus umgangssprachliche Formulierungen vor. Zudem macht Sieber (1998, S. 41) darauf aufmerksam, dass an mehreren Stellen innerhalb eines Satzes ein Registerwechsel zu beobachten sei (z.B. «Der Mönch, die ganze Zeit im Kloster verbringend, besitzt ein sogenanntes ,babyface', völlig faltenfrei.») und dass neben Sätzen mit «deutlicher syntaktischer 
Komposition» wenig geplante Konstruktionen vorkommen würden. Auch Ellipsen, Ausklammerungen, Anakoluthe und Parenthesen würden, so Sieber (1998), im Parlando-Schreiben "beinahe selbstverständlich in die Texte eingebaut»(S. 44). All dies sind Merkmale, wie sie auch in SMS-Schreiben vorkommen. Ein Beispiel für die Ellipse eines Objektpronomens wurde weiter oben schon genannt, weitere Belege finden sich in der SMS «Weißt du tel. von mech. Werkstatt?» in (6), wo an zwei Stellen der Artikel fehlt. Solche Auslassungen können dazu dienen, Zeichen einzusparen, Zeit und Tippaufwand zu reduzieren; sie sind aber auch schon zu einem Stilmittel in der SMS geworden. Auch in dem kurzen Text in (6) hätte der Schreiber durchaus noch Zeichen zur Verfügung gehabt, er hätte die Artikel setzen und auch die abgekürzten Wörter ausschreiben können, ohne dass eine weitere SMS erforderlich gewesen wäre. Er tut dies dennoch nicht. Ein Grund mag auch der sein, dass ein solch ökonomisches Schreiben als SMS-typisch gilt und man davon ausgehen kann, dass es nicht als Mangel angelastet wird, wenn man auf das Setzen des Artikels verzichtet, Wörter abkürzt, Substantive durchgängig kleinschreibt oder Satzzeichen weglässt. Im Parlando-Schreiben finden sich diese graphischen Besonderheiten nicht. Zwar konstatiert Sieber (1998) eine "Unbekümmertheit oder Unachtsamkeit» (S. 45) in der Interpunktion, die sich darin zeige, dass Satzschlusszeichen vergessen oder Kommas nicht gesetzt werden, obwohl an anderer Stelle die Kommasetzung völlig korrekt sei. Andere SMS-typische Schreibweisen wie Abkürzungen und Akronyme kommen in den Maturarbeiten jedoch nicht vor. Ohnehin scheinen die Orthographiefehler in den ParlandoTexten keine nennenswerte Rolle zu spielen. So erwähnt Sieber (1998), dass immerhin 82\% der 6'683 Sätze in seinem Korpus «interpunktionsmässig korrekt geschrieben wurden» (S. 45).

Ein weiterer, grundsätzlicher Aspekt ist an dieser Stelle noch zu betonen: Weder das Auslassen von Artikeln noch das Abkürzen von Wörtern oder die durchgängige Kleinschreibung von Substantiven sind Merkmale konzeptioneller Mündlichkeit. Das Auslassen von Artikelwörtern, aber auch von Präpositionen, wie es in Äusserungen vom Typus Gehen wir Bahnhof? der Fall ist, mag typisch für das so genannte ethnolektale Deutsch sein (Dürscheid \& Spitzmüller, 2006), das in der Schweiz auch als «Balkandeutsch» bezeichnet wird; es ist aber nicht typisch für einen konzeptionell mündlichen Sprachgebrauch, wie ihn Koch und Oesterreicher (1994) charakterisieren. In den Anfängen der SMS-Kommunikation traten diese Auslassungen nur deshalb auf, um Zeichen und Tippaufwand zu sparen, sie waren also den technologischen Gegebenheiten des Handyschreibens geschuldet. Das trifft ebenso auf die oben genannten graphischen Merkmale in SMS zu, die sich auch in den Beispielen (4) und (5) zeigen: Wörter werden als Akronyme gesetzt (z.B. tp für Treffpunkt), Satzzeichen fehlen, Substantive werden klein-, andere Wörter fälschlich grossgeschrieben. So beginnt in der SMS unter (5) das Wort, das dem Abkürzungspunkt folgt, mit einem Grossbuchstaben («ca. Noch»). Das hängt damit zusammen, dass die 
Handy-Software T9 den Punkt als Satzschlusszeichen interpretiert und deshalb das folgende Wort automatisch grossschreibt. Alle diese Merkmale betreffen die Art und Weise ihrer Verschriftung, nicht den sprachlichen Ausdruck. Sie dürfen also nicht zur konzeptionellen Mündlichkeit gezählt werden.

Werfen wir an dieser Stelle noch einen Blick auf die Chatkommunikation. Auch hier treten graphostilistische Besonderheiten (z.B. Smileys) sowie Merkmale konzeptioneller Mündlichkeit (z.B. Gesprächspartikeln) auf. Das verwundert nicht: Wie weiter oben schon gesagt, liegt im Chat eine dialogische Situation vor. Es ist also nicht nur - wie in der SMS-Kommunikation - so, dass die an einem Chat Beteiligten davon ausgehen, dass die/der andere bald antworten wird; die Kommunikation kommt nur dann zustande, wenn sich alle Beteiligten im selben, virtuellen, Raum befinden und gleichzeitig an der Tastatur präsent sind. Wer einen Chatbeitrag schreibt, weiss also, dass er/sie in kürzester Zeit eine Antwort bekommen wird - es sei denn, die Tastatur wurde von der anderen Person kurzfristig verlassen. Auf die Wiedergabe eines Chatmitschnitts verzichte ich hier aus Platzgründen, stattdessen sei auf den Beitrag von Kilian (2006) verwiesen, in dem Beispiele aus einem deutschen Chatraum präsentiert werden und die hier auftretenden, typischen sprachlichen Merkmale beschrieben werden. So weist Kilian (2006) darauf hin, dass sich in diesem Chat die gesprochene Umgangssprache im Schriftbild widerspiegeln würde, was sich beispielsweise an Verschmelzungen von finitem Verb und Personalpronomen zeige (z.B. so issses, haste). In Deutschschweizer Chaträumen kommt ein solches Schreiben auch vor; hinzu kommt, dass es hier häufig ein Dialekt ist, der auf diese Weise phonetisch verschriftet wird. So fragt ein Zürcher Student in einem mir vorliegenden Chatmitschnitt seinen Kollegen: «hesch du ez alli prüefige dure?», woraufhin der andere «nei» antwortet und dann auf Nachfrage ergänzt: «am mäntig di letscht».

Ein solch dialektales Schreiben ist, wie ich an anderer Stelle gezeigt habe (Dürscheid, Wagner \& Brommer, 2010), typisch für die private Chatkommunikation in der Deutschschweiz. Die beiden Studenten schreiben, wie sie miteinander sprechen würden. Der Grund liegt auf der Hand: Ihr Schreiben ist dialogisch, sie sind miteinander vertraut, der Schreibanlass ist privater Natur. Ein Gespräch ist es dennoch nicht, der eine kann den anderen nicht unterbrechen, die Kommunikation erfolgt schriftlich, nicht (medial) mündlich, die Beiträge werden aufgezeichnet und sind, solange der Nachrichtenaustausch andauert, jederzeit nachlesbar. Die Kommunikation ist also weder synchron noch flüchtig. Letzteres wäre nur dann der Fall, wenn über das Internet tatsächlich miteinander gesprochen würde, wenn also z.B. ein Gespräch via Skype geführt wird oder man sich in einem Online-Spiel in einem Voice-Chat austauscht. Nur dann kann man auch streng genommen von Mündlichkeit in den neuen Medien sprechen, in allen anderen Fällen sollte (nur) von konzeptioneller Mündlichkeit die Rede sein. 


\section{Ausblick: von den neuen Medien zur Schreib- kompetenz}

Im vorliegenden Beitrag wurde gezeigt, dass es in der Diskussion um aktuelle Entwicklungen in der Mündlichkeit wichtig ist, sich zunächst klar zu machen, welches Konzept hinter dem Wort Mündlichkeit steht. Auch wurde deutlich, dass das Schreiben in den neuen Medien häufig zwar ein dialogisches Schreiben ist, dass es aber weder ein Gespräch ist noch dem entspricht, was Peter Sieber in seinen Untersuchungen zu Maturaufsätzen als Parlando bezeichnet hat. Am nächsten kommt man dem privaten, dialogischen Schreiben via Computer und Handy, wenn man sich fragt, inwieweit es konzeptionell mündlich ist. Dabei wird man feststellen, dass ein privater Chat z.B. nicht per se als konzeptionell mündlich bezeichnet werden kann, sondern es möglicherweise hierfür typische Merkmale gibt, die häufiger auftreten, und andere, die nicht vorkommen. Auch können nicht alle Auffälligkeiten in SMS- und dialogischen Internettexten damit erfasst werden. Wie weiter oben gezeigt wurde, liegen die graphostilistischen Merkmale (z.B. Akronyme) nicht auf der Ebene konzeptioneller Mündlichkeit, und auch nicht alle auffälligen syntaktischen Merkmale (z.B. die Auslassung von Artikeln und Präpositionen) sind konzeptionell mündlich.

Damit habe ich die wichtigsten Aspekte zum Thema Parlando, Mündlichkeit und neue Medien zusammengefasst, nun sei abschliessend noch ein Blick auf die Schreibkompetenz von Schülerinnen und Schülern geworfen. Eine Frage wird in diesem Zusammenhang immer wieder gestellt: Kann es sein, dass einige der SMS- und internetspezifischen Merkmale sich auch im Schreiben von Schultexten finden, zumal es v.a. Jugendliche sind, die in ihrer Freizeit diese neuen Kommunikationsformen nutzen, Nachrichten in Facebook schreiben, sich an Online-Spielen beteiligen und vieles mehr? Ein vom SNF gefördertes Forschungsprojekt zum Thema «Schreibkompetenz und neue Medien», das unter meiner Leitung stand, ging dieser Frage in enger Zusammenarbeit mit Kantonsschulen, Sekundarschulen und Berufsschulen im Kanton Zürich und Zug nach. Verglichen wurden über 1'000 Freizeittexte mit fast ebenso vielen Schultexten; zusätzlich wurden die an der Untersuchung beteiligten 754 Schülerinnen und Schüler nach ihren Schreib- und Lesegewohnheiten in der Freizeit befragt. Auf die Ergebnisse dieser Studie kann ich hier nicht detailliert eingehen (Dürscheid et al., 2010), es sei nur so viel gesagt: Es gibt keine Evidenz dafür, dass das private, dialogische Schreiben in den neuen Medien einen Niederschlag in den Schultexten findet. Zwar kann es vereinzelt vorkommen, dass ein Smiley gesetzt wird, doch die meisten Merkmale, die typisch für das Schreiben in den neuen Medien sind und weiter oben erwähnt wurden (z.B. Abkürzungen, durchgängige Substantivkleinschreibungen, durchgängige Grossschreibung, Akronyme, Ellipsen, Anakoluthe), finden sich in den Schultexten nicht. Die Schülerinnen und Schüler wissen die beiden Schreibwelten, die private und die schulische, zu trennen. Das heisst nicht, dass die Jugendlichen immer den 
schulischen Anforderungen entsprechend schreiben; die Fehler, die in den Schultexten auftreten, sind aber nicht kausal darauf zurückzuführen, dass sie in ihrer Freizeit chatten, SMS verschicken oder Nachrichten in Facebook schreiben.

\section{Anmerkungen}

1 Informationen dazu finden sich unter www.jugenddebattiert.ch (Zugriff am 12.06.2011). Hier sei zum Ablauf der Debatten nur so viel gesagt: Vier Schülerinnen und Schüler debattieren eine Streitfrage (z.B. «Soll der Bundesrat vom Volk gewählt werden?»). Im Anschluss daran werden die Beiträge auf der Basis der folgenden Kriterien bewertet: Ausdrucksfähigkeit, Überzeugungskraft, Gesprächsfähigkeit und Sachkenntnis.

2 So schreibt Eriksson (2009): «Die Beschreibung, Erfassung, und Beurteilung mündlicher Sprachkompetenzen hat im Bereich der Schulsprache, ganz anders als im Fremdsprachenunterricht, noch wenig Tradition.» (S. 120). Ein Grund hierfür ist vermutlich, dass man lange Zeit der Auffassung war, Schülerinnen und Schüler mit Deutsch als Erstsprache beherrschten diesen Bereich ohnehin. Diese Überlegung spielte in der Linguistik keine Rolle, hier dominierte die Schriftlichkeit, weil grammatische Analysen nur an geschriebenem Material vorgenommen werden konnten, solange man keine Möglichkeiten zur Tonaufzeichnung gesprochener Sprache hatte.

3 In der Deutschschweiz kommt hinzu, dass die Schriftsprache als Synonym für Standardsprache steht, obwohl Letztere ja nicht nur geschrieben, sondern, wenn auch weitaus seltener, gesprochen wird. Dass dennoch eine solche Gleichsetzung vorgenommen wird, zeigt, wie stark die mediale Diglossie (geschrieben wird Hochdeutsch, gesprochen wird Dialekt) im Bewusstsein verankert ist.

4 Die hier vorgenommene Unterscheidung gilt nur noch für den prototypischen Fall. Heute führt das Smartphone die Nutzungsmöglichkeiten von Computer und Handy in einem kleinen, mobilen Gerät zusammen.

5 An dieser Stelle sei angemerkt, dass die Thematisierung eigener Erfahrungen in der Deutschmatur gelegentlich sogar explizit verlangt wird. So heisst es in einer Prüfungsaufgabe zur Interpretation eines Gedichtes von Bertolt Brecht, man solle das Thema aufgrund der "persönlichen Erfahrung» reflektieren. Solche Aufforderungen tragen zu einem Parlando-Schreiben bei. Als Hochschullehrerin, die von ihren Studierenden einen argumentativ-wissenschaftlichen Schreibstil erwartet, stehe ich dem kritisch gegenüber.

\section{Literatur}

Becker-Mrotzek, M. (Hrsg.). (2009). Mündliche Kommunikation und Gesprächsdidaktik. Baltmannsweiler: Schneider Verlag Hohengehren.

Behaghel, O. (1899). Geschriebenes Deutsch und gesprochenes Deutsch. Abgedruckt in O. Behaghel (1927), Von deutscher Sprache. Aufsätze, Vorträge und Plaudereien (S. 11-34). Lahr: Moritz Schauenburg.

Brinker, K. \& Sager, S. F. (1973/2010). Linguistische Gesprächsanalyse: Eine Einführung (5., neu bearbeitete Auflage). Berlin: Erich Schmidt Verlag.

Duden. (2009). Die Grammatik. Unentbehrlich fïr richtiges Deutsch (Bd. 4., 8. Auflage). Mannheim: Dudenverlag.

Dürscheid, Ch. (2006). Einführung in die Schriftlinguistik (Ergänzt um ein Kapitel zur Typographie von Jürgen Spitzmüller; 3., überarbeitete und ergänzte Auflage). Göttingen: Vandenhoeck \& Ruprecht.

Dürscheid, Ch. \& Spitzmüller, J. (2006). Jugendlicher Sprachgebrauch in der Schweiz: Eine Zwischenbilanz. In Ch. Dürscheid \& J. Spitzmüller (Hrsg.), Zwischentöne. Zur Sprache der 
Jugend in der Deutschschweiz (S. 13-48). Zürich: Verlag Neue Zürcher Zeitung.

Dürscheid, Ch., Wagner, F. \& Brommer, S. (2010). Wie Jugendliche schreiben. Schreibkompetenz und neue Medien (Mit einem Beitrag von Saskia Waibel). Berlin: De Gruyter.

Eriksson, B. (2009). Bildungsstandards - Mündliche Kommunikation. In M. BeckerMrotzek (Hrsg.), Mündliche Kommunikation und Gesprächsdidaktik (S. 116-128). Baltmannsweiler: Schneider Verlag Hohengehren.

Eriksson, B. (2010). Ich spreche schulisch! Mündliche Sprachkompetenz - Schule und Schriftlichkeit. Schulinfo Zug. Thema: Sprechen (Hrsg. von der Direktion für Bildung und Kultur. Kanton Zug), 5-8.

Kilian, J. (2006). Standardnorm versus Parlando in Schüler/innen-Chats. Der Deutschunterricht, 5, 74-83.

Koch, P. \& Oesterreicher, W. (1994). Schriftlichkeit und Sprache. In H. Günther \& O. Ludwig (Hrsg.), Schrift und Schriftlichkeit. Writing and its use. Ein interdisziplinäres Handbuch internationaler Forschung. An interdisciplinary handbook of international research (1. Halbband) (S. 587-604). Berlin: De Gruyter.

Koch, P. \& Oesterreicher, W. (2007). Schriftlichkeit und kommunikative Distanz. Zeitschrift für germanistische Linguistik, 35 (3), 346-375.

Krelle, M. \& Spiegel, C. (Hrsg.). (2009). Sprechen und Kommunizieren. Entwicklungsperspektiven, Diagnosemöglichkeiten und Lernszenarien. Baltmannsweiler: Schneider Verlag Hohengehren.

Ong, W. (1982/1987). Orality and literality. The technologizing of the word. London: Methuen \& Co. Deutsche Übersetzung: Oralität und Literalität. Die Technologisierung des Wortes. Opladen: Westdeutscher Verlag.

Quasthoff, U. (2009). Entwicklung der mündlichen Kommunikationskompetenz. In M. Becker-Mrotzek (Hrsg.), Mündliche Kommunikation und Gesprächsdidaktik (S. 84100). Baltmannsweiler: Schneider Verlag Hohengehren.

Radisch, I. (2010). Zur Lage der Literatur. ZEIT, Ressort: Kultur. Zugriff am 22.01.2011 unter http://www.zeit.de/2010/40/Gegenwartsliteratur

Roth, K. S. (2006). Die Jugend ,zum Reden bringen'? Sprachwissenschaftliche Perspektiven auf die Rhetorik im Muttersprachunterricht. In Ch. Dürscheid \& J. Spitzmüller (Hrsg.), Perspektiven der Jugendsprachforschung/Trends and developments in youth language research (S. 73-86). Frankfurt am Main: Peter Lang.

Schlobinski, P. (Hrsg.). (2006). Von ${ }^{*} b d l^{*}$ bis ${ }^{*}$ culser* Sprache und Kommunikation in den neuen Medien. Mannheim: Bibliographisches Institut.

Schwitalla, J. (2006). Gesprochenes Deutsch. Eine Einführung (3., neu bearbeitete Auflage). Berlin: Erich Schmidt Verlag.

Sieber, P. (1998). Parlando in Texten. Zur Veränderung kommunikativer Grundmuster in der Schriftlichkeit. Tübingen: Niemeyer.

Sieber, P. (2008). Kriterien der Textbewertung am Beispiel von Parlando. In N. Janich (Hrsg.), Textlinguistik. 15 Einführungen (S. 271-289). Tübingen: Narr.

Schlagworte: Parlando, Mündlichkeit, Schreibstil, neue Medien, SMS-Kommunikation 


\section{Parlando, oralité et nouveaux médias. Quelques considérations d'un point de vue linguistique}

Résumé

L'article débute par une réflexion à propos des termes konzeptionell mündlich ('conceptionnellement oral'), medial mündlich ('médialement oral'), et gesprochensprachlich ('langage parlé') et en décrivant les critères qui caractérisent les textes relevant du style dénommé parlando. Cette dénomination a été proposée pour saisir ce que certains considèrent comme un nouveau style d'écriture, basé sur des traits qu'on trouve normalement dans le langage parlé. La question traitée ici est de savoir s'il est justifié de considérer la communication écrite privée typique des nouveaux médias (e-mails, sms, chats...) comme des textes oraux représentés dans le code écrit et, dès lors, de la rapprocher du langage parlé.

L'article montre que des traits d'un style conceptionnel oral sont certes fréquents dans ces textes mais que ceux-ci ne peuvent pas, pour autant, être considérés comme équivalents à ce que Sieber (1998) a nommé, en examinant des travaux de maturité, le style parlando. Les deux niveaux doivent être précisément distingués et l'idée d'oralité dans les nouveaux médias ne doit être utilisée que pour décrire des formes de communication qui sont effectivement orales, telles que les appels via Skype sur Internet. Dans tous les autres cas, et cela constitue l'argumentation finale du texte, seul le terme d'oralité conceptionnelle est applicable tandis que les expressions d'oralité et de parlando doivent être évitées.

Mots clés: Parlando, oralité, style écrit, nouveaux médias, textos

\section{Parlando, oralità e media. Riflessioni da una prospettiva linguistica}

\section{Riassunto}

Questo articolo muove da una discussione e distinzione dei termini konzeptionell mündlich ('concettualmente orale'), medial mündlich ('orale mediale'), e gesprochensprachlich ('linguaggio parlato'), che vengono spesso riuniti sotto l'etichetta del cosiddetto stile parlando a indicare un nuovo stile di scrittura orientato al parlato (cf. Sieber, 1998).

Viene poi posta la domanda centrale: se sia corretto caratterizzare come oralità scritta la comunicazione testuale privata attraverso i nuovi media (ad es., email, SMS, chat), e dunque considerarla vicina al linguaggio parlato. L'analisi porta su testi di messaggi SMS e chat.

L'articolo mostra come questi testi di scrittura privata mostrino molti tratti tipici dell'oralità, che in linguistica vengono fatti risalire all'etichetta konzep- 
tionell mündlich; allo stesso tempo però, viene mostrato che questi stessi testi non possono essere considerati uguali a ciò che Peter Sieber chiama stile parlando nella sua analisi dei lavori di maturità. I due livelli vanno ben distinti, e il termine oralità nei nuovi media può essere solo usato quando ci si riferisce a reali pratiche orali (ad es., telefonate via internet o via Skype). In tutti gli altri casi, e questa è la conclusione dell'articolo, è più corretto parlare di oralità concettuale, ma non di oralità nè di parlando.

Parole chiave: Parlando, oralità, stili di scrittura, nuovi media, SMS

\section{Parlando style, orality and new media. Some remarks from a linguistic point of view}

\section{Abstract}

The paper starts with a reflection on the terms konzeptionell mündlich («conceptually oral»), medial mündlich («medially oral»), and gesprochensprachlich («spoken language») by laying out the criteria that characterize texts in the so called parlando style (cf. Sieber, 1998), which is often seen as a defining marker for a new writing style relying on features normally found in spoken language. Then, focusing on text messaging and chat communication, I will question the often found description of text-based communication in the new media (e.g. email, SMS, chat) as «oral» texts represented in the written code and of aligning it with spoken language. I will show that features of a conceptually oral style are frequent in these texts, but that they still cannot be set equal to what Peter Sieber calls parlando style when investigating final papers from highschools. The two levels have to be differentiated diligently and the term orality in the new media should only be used to describe communication forms that are actually oral, such as Internet calls via Skype. In all other cases, and this will be my final argumentation, only the term conceptually oral style is applicable while the expressions orality and parlando should be avoided.

Key words: Parlando style, orality, writing style, new media, text messaging 